\title{
A Simple Preparation of Stationary Phase of Alkylamide Reversed-Phase Liquid Chromatograph for the Separation of Basic Substances
}

\author{
Xiaojia Huang, Junde Wang, ${ }^{\dagger}$ and Xueliang LIU \\ Dalian Institute of Chemical Physics, Chinese Academy of Sciences, \\ 161 Zhongshan Road, Dalian, 116012, People's Republic of China
}

\begin{abstract}
A simple preparation process of alkylamide phase for reversed-phase HPLC (RP-HPLC) is described. The process includes aminopropyltrimethoxysilane firstly reacted with octanoyl chloride, then the intermediate was coupled onto porous silica. The resultant bonded silica has a reproducible ligand surface concentration and homogenous bonded ligand distribution on the porous silica. Characterization of prepared packing was carried out with elemental analysis, solid-state ${ }^{13} \mathrm{C}$ NMR and Fourier transform infrared (FT-IR). Chromatographic evaluations were carried out by using a mixture of organic compounds including acidic, basic and neutral analytes under methanol/water as binary mobile phase. The results showed that the stationary phase have excellent chromatographic properties and can be efficiently used for the separation of basic compounds.
\end{abstract}

(Received June 12, 2001; Accepted October 10, 2001)

Basic compounds, e.g. most pharmaceuticals and some peptides, are difficult to be separated or purified on silica-based reversed phase materials for liquid chromatography because of undesired adsorption of the basic nitrogen containing groups to acidic sites on the silica surface. Acceptable separations have been achieved using high-purity silica, ${ }^{1}$ silica end-capped with short-chain alkyl silanes, ${ }^{2}$ and mobile-phase conditions that suppress analyte/silanol interactions. ${ }^{3}$ Another solution is to use a reversed-phase silica possessing a ligand with an internal polar functional group. ${ }^{4,5}$ The internal polar group seems to weaken the interaction between basic analytes and residual silanol groups on the silica surface, so the process can be used to separate basic substance effectively. Silica packings of this type are commonly prepared via two steps. ${ }^{6,7}$ The amino phase is formed in the first step and further modified in the second step by attaching an alkyl chain via reaction of an acid chloride with the amino ligands. This methodology suffers from the difficulty in modifying the silica reproducibly over two successive reactions. At the same time, a poorly defined mixture of derivatized and underivatized amino groups may exist simultaneously on the silica surface as outlined in Fig. 1(a), and the underivatized aminopropyl ligands possess aminoexchange properties at low $\mathrm{pH}$ which can negatively influence their chromatographic behavior for acidic compounds. O'Gara and co-workers described a new reaction process to synthesize silica bonded phase containing internal polar carbamate functional groups. ${ }^{8}$ It is reported that the final products possess homogeneous bonded ligands and have reproducible ligand surface concentrations. But there are two shortcomings in this methodology. Firstly, as outlined in Fig. 1(b), three reaction steps were involved in the preparation process, so more exhaustive work is needed. Secondly, the prices of the reaction

† To whom correspondence should be addressed.

E-mail: wjd@dicp.ac.cn materials are more expensive than those of conventional reaction.

In this report, a simple process of alkylamide phase for RPHPLC is described. The process includes aminopropyltrimethoxysilane firstly reacted with octanoyl chloride by employing pyridine as catalyst; then the intermediate mixture was filtered and the filtrate was coupled directly onto porous silica. In contrast to the conventional two stages reaction, this new method omits the step of washing the intermediate product with organic solvents, which are noxious to the human body and to the environment. Furthermore, the distribution of bonded ligands in the final stationary phase is homogenous and the reproducibility is satisfactory. At the same time, the price of reaction materials in this process is much lower than that reported by O'Gara.

\section{Experimental}

\section{Chemical and solvents}

Octanoyl chloride (99\%) was purchased from Fluka. $\gamma$ Aminopropyltrimethoxysilane (APS) was from Aldrich. Endcapped $\mathrm{C}_{8}$ bonded phase (surface concentration: $3.6 \mu \mathrm{mol} / \mathrm{m}^{2}$ ) and Kromasil silica (mean particle diameter, $5 \mu \mathrm{m}$; specific surface area, $340 \mathrm{~m}^{2} \mathrm{~g}^{-1}$; mean pore diameter, $10 \mathrm{~nm}$ ) were purchased from Akzo Nobel (Bohus, Sweden). HPLC grade methanol was from Yuwang Chemical Factory (Shandong, China). Toluene, pyridine and other reagents utilized were all of analytical-reagent grade. Double distilled water was used.

\section{Apparatus}

HPLC separations were carried out at ambient temperature on a system consisting of an LC-10AD HPLC pump (Shimadzu, Japan), SPD-10A UV/VIS spectrophotometer detector (Shimadzu, Japan) and Rheodyne 7125 injection valve (Cotati, 
(a)

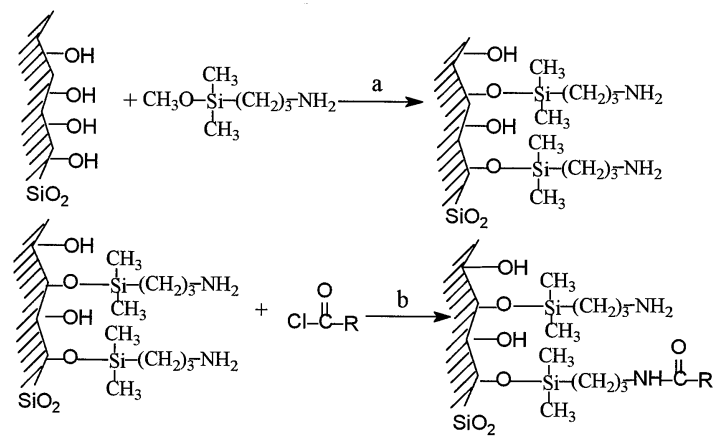

(b)

$$
\begin{aligned}
& \mathrm{CH}_{2}=\mathrm{CH}-\mathrm{CH}_{2} \mathrm{O}-\stackrel{\mathrm{O}}{\mathrm{C}}-\mathrm{Cl}
\end{aligned}
$$

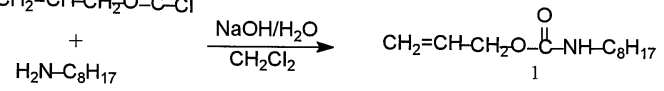

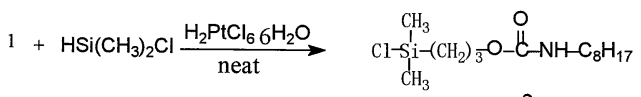

$$
\begin{aligned}
& 2+\sum_{-\mathrm{OH}}^{\mathrm{OH}}
\end{aligned}
$$

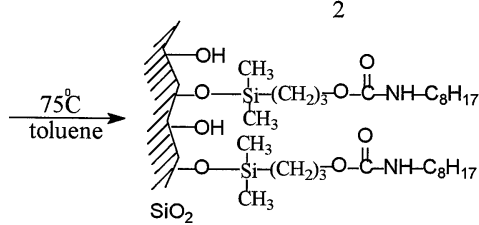

(c)

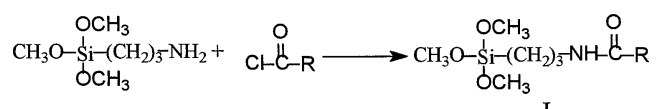

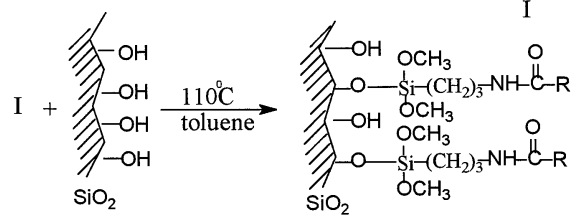

Fig. 1 (a) The conventional two-step methodology for the synthesis of amide-bonded phase. ${ }^{8}$ (b) The preparation route of $\mathrm{C}_{8}$ HPLC packing with an internal carbamate functional group reported by O'Gara. ${ }^{8}$ (c) The simple preparation methodology in this research. Reproduced by permission from Ref. 8 .

CA, USA). JS-3000 chromatographic workstation (Dalian, China) was used for chromatogram recording and data processing.

Carbon, hydrogen and nitrogen percentages for the new stationary phase were determined on a Carlo Erba Model 1106 analyzer (Italy). The infrared spectra were acquired on an FTIR 170-SX infrared spectrophotometer (Nicolet, USA). The solid state ${ }^{13} \mathrm{C}$ CP-MAS-NMR spectra of the bonded silica were obtained on a Bruker MSL-300 Spectrometer (Billerica, MA, USA).

\section{Pretreatment of silica gel}

Twenty grams of silica gel were added to a round-bottom flask containing $200 \mathrm{~mL}$ of $20 \%$ hydrochloric acid and the mixture was heated to reflux for $4 \mathrm{~h}$. The treated silica gel was filtered out, washed several times successively with water and acetone, then dried in a vacuum oven at $100^{\circ} \mathrm{C}$ for $12 \mathrm{~h}$. The dried silica was kept in a vacuum desiccator.

\section{Preparation of bonded phase}

The synthesis route was shown in Fig. 1(c). APS (10 mL) and dried pyridine $(3.5 \mathrm{~mL})$ were dissolved in $30 \mathrm{ml}$ of dried toluene in a three-neck round-bottom flask, then a mixture of $7.3 \mathrm{~mL}$ of octanoyl chloride (the molar ratio with APS was 1:1) in $20 \mathrm{~mL}$
Table 1 Elemental analysis of AOBP

\begin{tabular}{ccccc}
\hline Batch & $\mathrm{C} \%$ & $\mathrm{H} \%$ & $\mathrm{~N} \%$ & Surface concentration $/ \mu \mathrm{mol} \mathrm{m}{ }^{-2}$ \\
\hline 1 & 11.69 & 1.83 & 1.97 & 2.98 \\
2 & 11.62 & 1.78 & 1.94 & 2.95 \\
3 & 11.77 & 1.85 & 1.99 & 3.00 \\
\hline
\end{tabular}

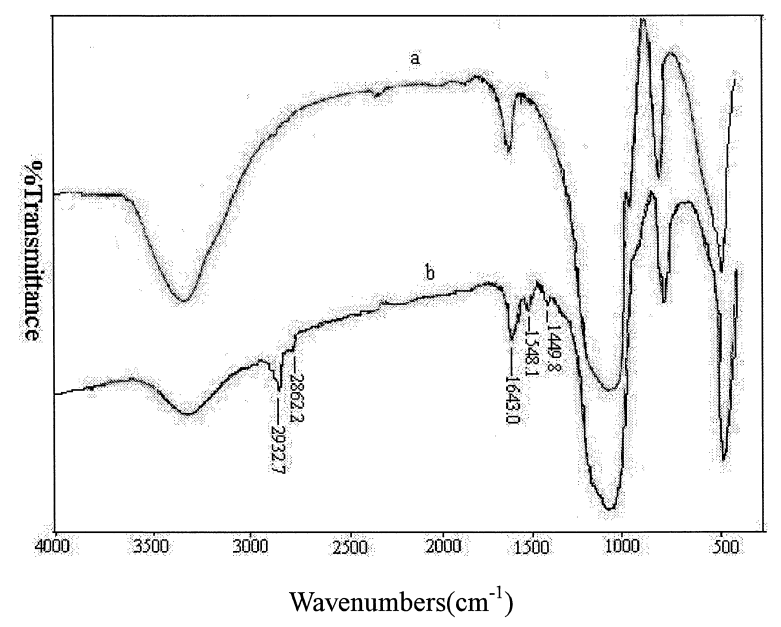

Fig. 2 FT-IR spectra of silica after treated by $20 \%$ hydrochloric acid (a), and the target silica bonded phase (b).

of dried toluene was added dropwisely while stirring; the flask was placed at room temperature for $6 \mathrm{~h}$ to ensure complete reaction. After reaction, the intermediate mixture was filtered out and the filtrate was stored in a desiccator for later use.

Dried silica gel $(5 \mathrm{~g})$ was placed in a $250 \mathrm{~mL}$ three-neck round-bottom flask and heated up to $150^{\circ} \mathrm{C}$ for $6 \mathrm{~h}$. Then the intermediate product in the above-mentioned filtrate was drawn into the flask by vacuum. After the slurry was agitated at refluxing temperature in an oil bath for $12-16 \mathrm{~h}$, the mixture was filtered out and washed with toluene and methanol several times respectively, then dried in vacuum at $100^{\circ} \mathrm{C}$ for $6 \mathrm{~h}$ to get the $\mathrm{C}_{8}$ amide-bonded phase (AOBP).

\section{Column packing}

Bonded silica was packed into $150 \times 4.6 \mathrm{~mm}$ stainless-steel columns using conventional high-pressure slurry techniques. Chloroform and ethanol were used as packing solvent and displacement solvent, respectively.

\section{Results and Discussion}

\section{Characterization of the packing}

The results of elemental analysis for three batches of the packings are listed in Table 1. It can be seen from the table that multiple bondings using the same batch of silane had identical surface concentrations within experimental error. That is to say, reproducible results can be achieved by the simple reaction route.

Figure 2 showed the FT-IR spectra of the final product and the starting silica of this packing. Compared with the spectrum of the starting silica gel, several new absorption bands have appeared in the spectrum of the final product. The peaks at $2932.7 \mathrm{~cm}^{-1}$ and $2862.2 \mathrm{~cm}^{-1}$ are asymmetric stretching vibration and symmetric stretching vibration of $-\mathrm{CH}_{2-}$, respectively; and the peak at $1449.8 \mathrm{~cm}^{-1}$ is assigned to the 

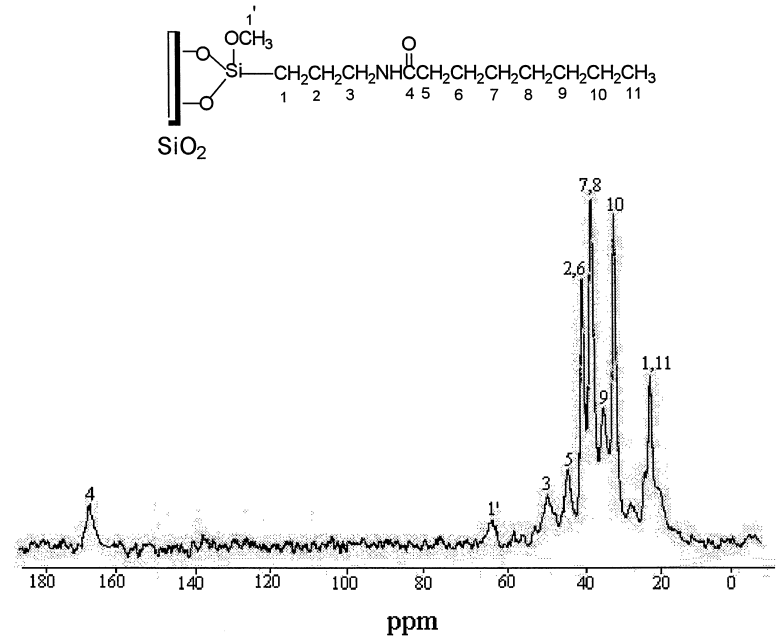

Fig. 3 Solid state ${ }^{13} \mathrm{C}$-CP-MAS NMR spectrum of the bonded phase prepared.

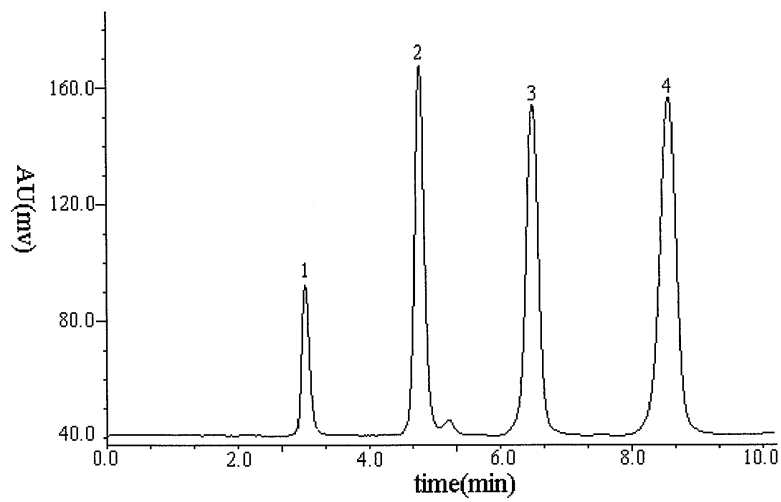

Fig. 4 The chromatogram of some aromatic compounds. Conditions: mobile phase, $75: 25(\mathrm{v} / \mathrm{v})$ methanol/water; flow rate, 0.8 $\mathrm{ml} / \mathrm{min}$; column temp., $25^{\circ} \mathrm{C}$; detection wavelength, $254 \mathrm{~nm}$. Peak No. 1, benzene; 2, naphthalene; 3 , diphenyl; 4, phenanthrene.

bending vibration of $-\mathrm{CH}_{2}-$; while the amide $\mathrm{I}\left(v_{\mathrm{C}=0}\right)$ and amide II $\left(\delta_{\mathrm{N}-\mathrm{H}}+v_{\mathrm{C}-\mathrm{N}}\right)$ appear at $1643.0 \mathrm{~cm}^{-1}$ and $1548.1 \mathrm{~cm}^{-1}$, respectively. There is no absorption peak for $-\mathrm{NH}_{2}$, which indicates that homogenous bonded ligands are formed and that no underivatized aminopropyl ligand exists in the final product.

The solid-state ${ }^{13} \mathrm{C}$ CP-MAS-NMR of the final product is shown in Fig. 3. It can be seen from the spectrum that the peak near $171 \mathrm{ppm}$ can be assigned to the carbonyl carbon 4. Peaks in the range of 12 to $42 \mathrm{ppm}$ indicate the successful bonding of target long-chain-alkyl group onto the phase.

In short, the data of elemental analysis and the spectra of FTIR and solid-state ${ }^{13} \mathrm{C} C \mathrm{CP}-\mathrm{MAS}-\mathrm{NMR}$ confirm the success of the procedure of preparation.

\section{Chromatographic evaluation}

Chromatographic evaluation of the new stationary phase was carried out by using only methanol-water as the mobile phase. The separations of some aromatic compounds, basic compounds and a mixture of acidic, neutral and basic analytes on the new stationary phase are shown in Figs. 4, 5, and 6. Figure 4 is the separation result of some aromatic compounds on the new stationary phase. It can be seen that the four solutes have high theoretical plate numbers and good peak shapes (e.g., Biphenyl, $\left.N=49470, A_{\mathrm{s}}=0.95\right)$, which demonstrate the good hydrophobic

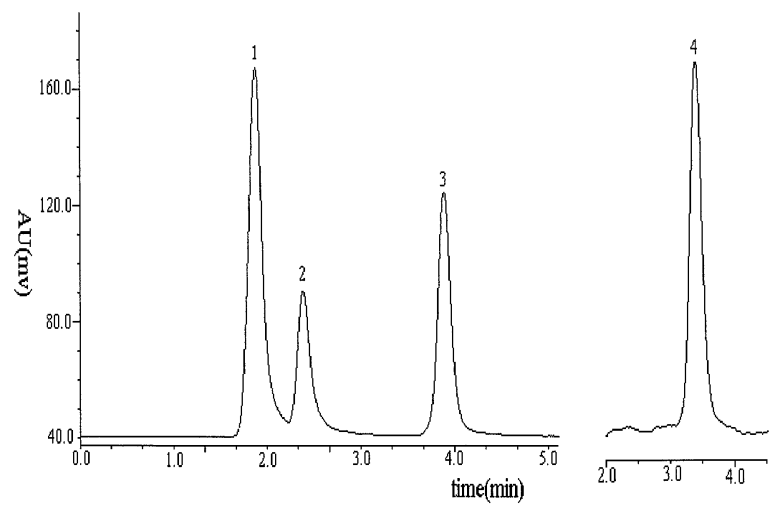

Fig. 5 The chromatogram of some basic compounds. Conditions: mobile phase, 65:35 (v/v) methanol/water; the others are the same as in Fig. 4. Peak No. 1, perphenazine; 2, papaverine; 3, aminophylline; 4 , nicotine.
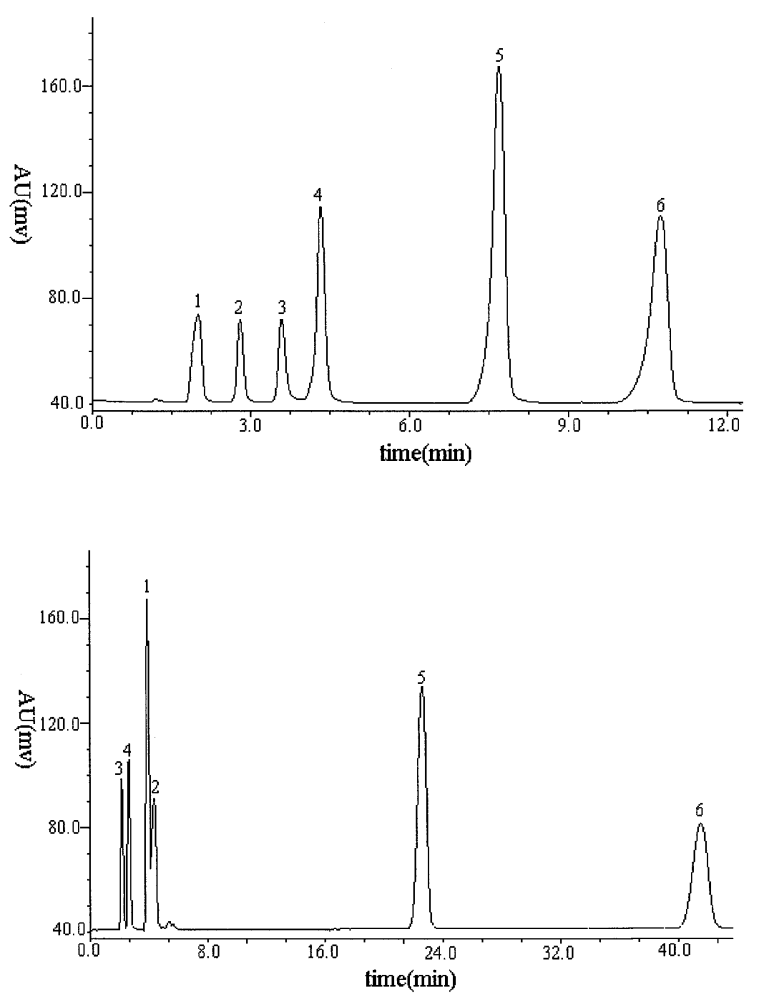

Fig. 6 Chromatograms of a mixture acidic, neutral and basic solutes by $\mathrm{C}_{8}$ amide-bonded phase (Top) and end-capped $\mathrm{C}_{8}$ bonded phase (Bottom). Mobile phase, 70:30 methanol/water; the others are the same as in Fig. 4. Peaks No.: 1, p-toluidine; 2, aminophylline; 3, pyrocatechol; 4 , phenol; 5 , toluene; 6 , ethylbenzene.

properties of the $\mathrm{C}_{8}$ amide-bonded phase.

Figure 5 shows the result of separation of some basic compounds including perphenazine, papaverine, aminophylline and nicotine under methanol and water as mobile phase. From the figure it can be seen that these basic solutes all have good peak shapes and high theoretical plate number (e.g., aminophylline $\left.N=28620, A_{\mathrm{s}}=1.11\right)$. The excellent chromatographic properties of the new packing are contributed to the existence of an internal polar functional group in the prepared stationary phase, which weaken the interaction between basic analytes and residual silanol groups on the silica 
Table 2 Comparison of chromatographic behaviors of AOBP and an end-capped $\mathrm{C}_{8}$ phase under methanol and water as mobile phase

\begin{tabular}{llc}
\hline \multirow{2}{*}{ Compound } & \multicolumn{1}{c}{ AOBP } & End-capped octyl \\
\cline { 2 - 3 } & \multicolumn{1}{c}{$k\left(A_{\mathrm{s}}\right)^{\mathrm{a}}$} & $k\left(A_{\mathrm{s}}\right)$ \\
\hline$p$-Toluidine & $0.031(0.93)$ & $0.19(1.37)$ \\
Aminophylline & $0.36(1.09)$ & $0.49(1.47)$ \\
Pyrocatechol & $0.74(1.2)$ & $0.68(1.03)$ \\
Phenol & $1.10(0.98)$ & $0.99(0.83)$ \\
Toluene & $2.74(0.86)$ & $12.8(0.90)$ \\
Ethylbenzene & $4.22(0.82)$ & $24.4(0.89)$ \\
\hline
\end{tabular}

a. Asymmetry factor.

The conditions are the same as in Fig. 6.

surface.

In order to further elucidate the efficiency of the amide bonded phase prepared in interacting with unreacted silanol groups on the silica surface, a comparison was made by separating a mixture which includes neutral, acidic and basic compounds under methanol and water as binary mobile phase between $\mathrm{AOBP}$ and an end-capped $\mathrm{C}_{8}$ bonded phase (Table 2 and Fig. 6). Under the test conditions, neutral and basic analytes had markedly lower capacity factors $(k)$ on the AOBP versus the end-capped $\mathrm{C}_{8}$ phase. The smaller capacity factors for basic compounds are attributed to weaker interaction with unbonded silanols due to the presence of the amide group, while smaller capacity factors for neutral analytes are attributed to the lower surface concentration of the AOBP versus the end-capped $\mathrm{C}_{8}$ phase, and hence less hydrophobic interaction between bondedphase and aromatic hydrocarbon analytes. ${ }^{9}$ The polar acidic compounds phenol and pyrocatechol were both slightly more retained on AOBP. That may be caused by an interaction between the phenolic proton and the highly polarized carbonyl oxygen atom of the amide group.

The AOBP was also compared to the end-capped $\mathrm{C}_{8}$ phase for hydrolytic stability under $\mathrm{pH} 2.5$ and $\mathrm{pH} 7.5$ buffered mobilephase conditions (65:35 methanol/buffer). As the plots in Fig. 7 show, no obvious difference in chemical stability was observed between the AOBP and the $\mathrm{C}_{8}$ bonded phase. Under $\mathrm{pH} 2.5$ buffer conditions, the hydrolytic stability of AOBP a little more stable than that of end-capped $\mathrm{C}_{8}$ phase. At $\mathrm{pH} 7.5$, the retention factor $k$ of ethylbenzoate decreased by $1.2 \%$ for AOBP and $1.0 \%$ for $\mathrm{C}_{8}$ bonded phase, respectively.

\section{Conclusions}

A simple procedure has been described for the preparation of a $\mathrm{C}_{8}$ amide-bonded phase. Compared to the conventional twostep preparation method, this methodology not only omits a step of washing procedure with organic solvents but also leads to

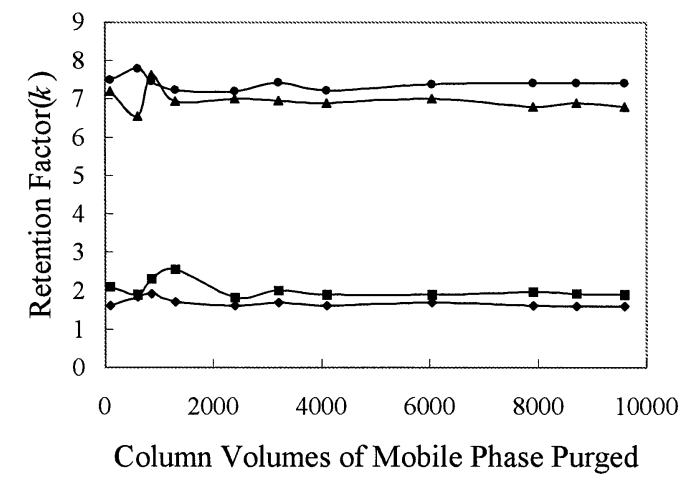

Fig. 7 Ethylbenzoate $k v s$. column volumes of mobile phase purged (mL) under $\mathrm{pH} 2.5$ and $\mathrm{pH} 7.5$ test conditions. (Top) End-capped $\mathrm{C}_{8}$ bonded phase, $--\mathrm{pH} 7.5,-\mathbf{\Delta}-\mathrm{pH} 2.5$. (Bottom) $\mathrm{C}_{8}$ amidebonded phase, $-\mathrm{pH} 7.5,-\mathrm{pH}$ 2.5. Mobile phase, 65:35 (v/v) methanol:0.02 mol/L disodium hydrogenphosphate-sodium dihydrogenphosphate buffer $(\mathrm{pH}=7.5) ; 65: 35(\mathrm{v} / \mathrm{v})$ methanol:0.02 $\mathrm{mol} / \mathrm{L}$ citric acid-disodium hydrogenphosphate buffer $(\mathrm{pH}=2.5)$; other conditions are the same as in Fig. 4.

attaining a reproducible and homogenous silica surface. Because of the existence of amide group in the final product, The AOBP may be useful in the separation of compounds that have undesirable interactions with silanols, or where a packing material with a selectivity different from that of a conventional $\mathrm{C}_{8}$ bonded phase is needed.

\section{References}

1. T. Hanai, M. Ohhira, and T. Tamura, $L C-G C, \mathbf{1 9 9 8 ,} 6,827$.

2. H. H. Freiser, M. P. Nowlan, and D. L. Gooding, J. Liq. Chromatogr., 1989, 12, 827.

3. M. A. Stadalius, J. S. Berus, and L. R. Synder, $L C-G C$, 1988, 6, 491.

4. J. J. Kirkland, J. W. Henderson, J. D. Martosella, B. A. Bidlingmeyer, J. Vasta-Rusell, and J. B. Adams, $L C-G C$, 1999, 17, 634.

5. C. P. Jaroniec, R. K.Gilpin, and M. Jaroniec, J. Chromatogr. A, 1998, 797, 103.

6. A. Nomura, J. Yamada, and K. Tsunoda, Anal. Sci., 1987, 3, 209.

7. B. Buszewski, P. Kasturi, R. K. Gilpin, M. E. Gargoda, and M. Jaroniece, Chromatographia, 1994, 39, 155.

8. J. E. O'Gara, B. A. Alden, T. H. Walter, J. S. Patersen, C. L. Niderlander, and U. D. Neue, Anal. Chem., 1995, 67, 3809.

9. H. Engelhardt and M. Jungheim, Chromatographia, 1990, $29,59$. 